\title{
Roles of Weight Functions to a Nonlocal Porous Medium Equation with Inner Absorption and Nonlocal Boundary Condition
}

\author{
Zhong Bo Fang, ${ }^{1}$ Jianyun Zhang, ${ }^{1}$ and Su-Cheol $\mathbf{Y i}^{2}$ \\ ${ }^{1}$ School of Mathematical Sciences, Ocean University of China, Qingdao 266100, China \\ 2 Department of Mathematics, Changwon National University, Changwon 641-773, Republic of Korea
}

Correspondence should be addressed to Zhong Bo Fang, fangzb7777@hotmail.com

Received 22 August 2012; Revised 24 October 2012; Accepted 7 November 2012

Academic Editor: Dragoş-Pătru Covei

Copyright (C) 2012 Zhong Bo Fang et al. This is an open access article distributed under the Creative Commons Attribution License, which permits unrestricted use, distribution, and reproduction in any medium, provided the original work is properly cited.

This work is concerned with an initial boundary value problem for a nonlocal porous medium equation with inner absorption and weighted nonlocal boundary condition. We obtain the roles of weight function on whether determining the blowup of nonnegative solutions or not and establish the precise blow-up rate estimates under some suitable condition.

\section{Introduction}

Our main interest lies in the following nonlocal porous medium equation with inner absorption term:

$$
u_{t}=\Delta u^{m}+u^{p} \int_{\Omega} u^{q}(y, t) d y-k u^{r}, \quad(x, t) \in \Omega \times(0,+\infty),
$$

subjected to weighted linear nonlocal boundary and initial conditions,

$$
\begin{gathered}
u(x, t)=\int_{\Omega} f(x, y) u(y, t) d y, \quad(x, t) \in \partial \Omega \times(0,+\infty), \\
u(x, 0)=u_{0}(x), \quad x \in \bar{\Omega}
\end{gathered}
$$

where $m>1, p \geq 0, q>0, p+q \geq 1, r \geq 1, k>0$, and $\Omega \subset R^{N}(N \geq 1)$ is a bounded domain with smooth boundary. The weight function $f(x, y) \not \equiv 0$ is a nonnegative continuous 
function defined on $\partial \Omega \times \bar{\Omega}$, and $\int_{\Omega} f(x, y) d y>0$ on $\partial \Omega$. The initial value $u_{0}(x) \in C^{2+\alpha}(\bar{\Omega})$ with $0<\alpha<1$ is a nonnegative continuous function satisfying the compatibility condition on $\partial \Omega$.

Many natural phenomena have been formulated as nonlocal diffusive equation (1.1), such as the model of non-Newton flux in the mechanics of fluid, the model of population, biological species, and filtration (we refer to $[1,2]$ and the references therein). For instance, in the diffusion system of some biological species with human-controlled distribution, $u(x, t), \Delta u^{m}, u^{p} \int_{\Omega} u^{q}(y, t) d y$, and $-k$ represent the density of the species, the mutation, the human-controlled distribution, and the decrement rate of biological species at location $x$ and time $t$, respectively. Due to the effect of spatial inhomogeneity, the arising of nonlocal term denotes that the evolution of the species at a point of space depends not only on the density of species in partial region but also on the total region (we refer to [3-5]). However, there are some important phenomena formulated as parabolic equations which are coupled with weighted nonlocal boundary conditions in mathematical models, such as thermoelasticity theory. In this case, the solution $u(x, t)$ describes entropy per volume of the material (we refer to $[6,7])$.

To motivate our work, let us recall some results of global and blow-up solutions to the initial boundary value problems with nonlocal terms or with nonlocal terms in boundary conditions (we refer to [8-16]). For the study of the initial boundary value problems for the parabolic equations with local terms which subject to the weighted nonlocal linear boundary condition (1.2), one can see [8-10]. For example, Friedman [8] studied the linear parabolic

$$
u_{t}-A u=0, \quad(x, t) \in \Omega \times(0, T),
$$

subjected to the nonlocal Dirichlet boundary condition (1.2), where $A$ is an elliptic operator,

$$
A=\sum_{i, j=1}^{n} a_{i, j}(x) \frac{\partial^{2}}{\partial x_{i} \partial x_{j}}+\sum_{i=1}^{n} b_{i}(x) \frac{\partial}{\partial x_{i}}+c(x), \quad c(x) \leq 0
$$

He proved that when $\int_{\Omega} f(x, y) d y \leq \rho<1$, the solution tends to 0 monotonously and exponentially as $t \rightarrow \infty$. With regard to more general discussions on initial boundary value problem for linear parabolic equation with nonlocal Neumann boundary condition, one can see [9] by Pao where the following problem was considered:

$$
\begin{gathered}
u_{t}-L u=g(x, u), \quad x \in \Omega, t>0, \\
B u=\int_{\Omega} f(x, y) u(y, t) d y, \quad x \in \partial \Omega, t>0, \\
u(x, 0)=u_{0}(x), \quad x \in \Omega,
\end{gathered}
$$

where

$$
L u=\sum_{i, j=1}^{n} a_{i j}(x) u_{x_{i} x_{j}}+\sum_{j=1}^{n} b_{j}(x) u_{x_{j}}, \quad B u=\alpha_{0} \frac{\partial u}{\partial n}+u .
$$


He studied the asymptotic behavior of solutions and found the influence of weight function on the existence of global and blow-up solutions. Wang et al. [10] studied porous medium equation with power form source term

$$
u_{t}=\Delta u^{m}+u^{p}, \quad(x, t) \in \Omega \times(0,+\infty),
$$

subjected to nonlocal boundary condition (1.2). By virtue of the method of upper-lower solutions, they obtained global existence, blow-up properties, and blow-up rate of solutions.

For the study of the initial boundary value problems for the parabolic equations with nonlocal terms which subjected to the weighted nonlocal linear boundary condition (1.2), we refer to [11-16]. Lin and Liu [11] considered the semilinear parabolic equation

$$
u_{t}=\Delta u+\int_{\Omega} g(u) d x, \quad(x, t) \in \Omega \times(0,+\infty),
$$

with nonlocal boundary condition (1.2). They established local existence, global existence, and blow-up properties of solutions. Moreover, they derived the uniform blow-up estimates for special $g(u)$ under suitable assumption; Cui and Yang [12] discussed the nonlocal slow diffusion equation

$$
u_{t}=\Delta u^{m}+a u^{p} \int_{\Omega} u^{q}(y, t) d y, \quad(x, t) \in \Omega \times(0,+\infty),
$$

and they built global existence, blow-up properties, and blow-up rate of solutions. For the system of equations, we refer readers to [13] and the references therein.

Recently, Wang et al. [14] studied the following semilinear parabolic equation with nonlocal sources and interior absorption term:

$$
u_{t}=\Delta u+\int_{\Omega} u^{q} d x-\alpha u^{r}, \quad(x, t) \in \Omega \times(0,+\infty),
$$

with weighted linear nonlocal boundary condition (1.2) and initial condition (1.3), where $q \geq 1, r \geq 1$, and $\alpha>0$. By using comparison principle and the method of upper-lower solutions, they got the following results.

(a) If $1 \leq q<r$, then the solution of the problem exists globally.

(b) If $q>r \geq 1$, the problem has solutions blowing up in finite time as well as global solutions. That is,

(i) if $\int_{\Omega} f(x, y) d y \leq 1$, and $u_{0}(x) \leq(\alpha /|\Omega|)^{1 /(r-q)}$, then the solution exists globally;

(ii) if $\int_{\Omega} f(x, y) d y>1$, and $u_{0}(x)>(\alpha /(|\Omega|-\alpha))^{1 / r}(|\Omega|>\alpha)$, then the solution blows up in finite time;

(iii) for any $f(x, y) \geq 0$, there exists $a_{2}>0$ such that the solution blows up in finite time provided that $u_{0}(x)>a_{2} \phi(x)$, where $\phi(x)$ is the corresponding normalized eigenfunction of $-\Delta$ with homogeneous Dirichlet boundary condition, and $\int_{\Omega} \phi(x) d x=1$. 
(c) If $q=r>1$.

(i) The solution blows up in finite time for any $f(x, y) \geq 0$ and large enough $u_{0}$.

(ii) If $\int_{\Omega} f(x, y) d y<1$, the solution exists globally for $u_{0}(x) \leq a_{1} \Phi(x)$ for some $a_{1}>0$, where $\Phi(x)$ solves the following problem:

$$
\begin{gathered}
-\Delta \Phi(x)=\delta_{0}, \quad x \in \Omega, \\
\Phi(x)=\int_{\Omega} f(x, y) d y, \quad x \in \partial \Omega,
\end{gathered}
$$

here $\delta_{0}$ is a positive constant such that $0 \leq \Phi(x) \leq 1$.

In addition, for the initial boundary value problem of (1.11) with weighted nonlinear boundary condition and Dirichlet boundary condition, we refer to $[15,16]$ and references therein, respectively.

The aim of this paper is to obtain the sufficient condition of global and blow-up solutions to problem (1.1)-(1.3) and to extend the results of the semilinear equation (1.11) to the quasilinear ones. The difficulty lies in finding the roles of weighted function in the boundary condition and the competitive relationship of nonlocal source and inner absorption on whether determining the blowup of solutions or not. Our detailed results are as follows.

Theorem 1.1. Suppose that $p+q>r$. If $\int_{\Omega} f(x, y) d y \geq 1$ for $x \in \partial \Omega$, and the initial data $u_{0}(x)>$ $(k /(|\Omega|-k))^{1 /(p+q)}(|\Omega|>k)$, then the solution of problem (1.1)-(1.3) blows up in finite time.

Remark 1.2. There may exist a global solution of problem (1.1)-(1.3) for small enough initial data under the condition of Theorem 1.1. Unfortunately, since the weight function satisfies the condition $\int_{\Omega} f(x, y) d y \geq 1$ on the boundary, we cannot construct a suitable supersolution of problem (1.1)-(1.3).

Theorem 1.3. Suppose that $p+q>r$, if $\int_{\Omega} f(x, y) d y \leq 1$ for $x \in \partial \Omega$, then the solution of problem (1.1)-(1.3) exists globally for the initial data $u_{0}(x)<(k /|\Omega|)^{1 /(p+q-r)}$. If $p+q \geq \max \{m, r\}$, then the solution of problem (1.1)-(1.3) blows up in finite time for large enough initial data and arbitrary $f(x, y)>0$.

Theorem 1.4. Suppose that $p+q<r$, then the solution of problem (1.1)-(1.3) exists globally for arbitrary $f(x, y)>0$.

Theorem 1.5. Suppose that $p+q=r$. $\int_{\Omega} f(x, y) \leq \rho<1$ for $x \in \partial \Omega$, where $\rho$ is a positive constant and $\rho<1$.

(1) If $m>p+q$, then every nonnegative solution of problem (1.1)-(1.3) exists globally.

(2) If $m=p+q$, then for $|\Omega|<k+\left(\delta / M_{1}\right)\left(1-\rho^{m}\right)$, the solution of problem (1.1)-(1.3) exists globally, where $\delta, M_{1}>0$ are as defined in (3.13).

(3) If $m<p+q$, the solution of problem (1.1)-(1.3) exists globally for sufficient small initial data while it blows up in finite time for large enough initial data.

In order to show blow-up rate estimate of the blow-up solution, we need the following assumptions on the initial data $u_{0}(x)$ :

$\left(C_{1}\right) \Delta u_{0}^{m}+u_{0}^{p} \int_{\Omega} u_{0}^{q}(y, t) d y-k u_{0}^{r}>0$, for $x \in \Omega ;$ 
$\left(C_{2}\right)$ there exists a constant $\delta^{\prime}>0$, such that

$$
\Delta u_{0}^{m}+u_{0}^{p} \int_{\Omega} u_{0}^{q}(y, t) d y-k u_{0}^{r}-\delta^{\prime} u_{0}^{p+q} \geq 0
$$

where $\delta^{\prime}$ will be determined later.

Theorem 1.6. Suppose that $p+q>\max \{m, r\}, \int_{\Omega} f(x, y) d y \leq 1$ for $x \in \partial \Omega$, and the initial data satisfies the conditions $\left(\mathrm{C}_{1}\right)-\left(\mathrm{C}_{2}\right)$, then

$$
c(T-t)^{-1 /(p+q-1)} \leq u(x, t) \leq C(T-t)^{-1 /(p+q-1)},
$$

where $c=[|\Omega|(p+q-1)]^{-1 /(p+q-1)}, C=[\delta(p+q-1) / m]^{-1 /(p+q-1)}$.

The rest of our paper is organized as follows. In Section 2, with the definitions of weak upper and lower solutions, we will give the comparison principle of problem (1.1)-(1.3), which is an important tool in our research. The proofs of results of global existence and blowup of solutions will be given in Section 3. And in Section 4, we will give the blow-up rate estimate of the blow-up solutions.

\section{Comparison Principle and Local Existence}

In this section, we establish a suitable comparison principle for problem (1.1)-(1.3). Let $Q_{T}=$ $\Omega \times(0, T), \bar{Q}_{T}=\bar{\Omega} \times(0, T)$, and $S_{T}=\partial \Omega \times(0, T)$. Firstly, we start with the precise definitions of upper solution and lower solution of problem (1.1)-(1.3).

Definition 2.1. Suppose that $\underline{u}(x, t) \in C^{2,1}\left(Q_{T}\right) \cap C\left(\bar{Q}_{T}\right)$ is nonnegative and satisfies

$$
\begin{gathered}
\underline{u}_{t} \leq \Delta \underline{u}^{m}+\underline{u}^{p} \int_{\Omega} \underline{u}^{q} d y-k \underline{u}^{r}, \quad(x, t) \in Q_{T}, \\
\underline{u}(x, t) \leq \int_{\Omega} f(x, y) \underline{u}(y, t) d y, \quad(x, t) \in S_{T}, \\
\underline{u}(x, 0) \leq \underline{u}_{0}(x), \quad x \in \Omega,
\end{gathered}
$$

then we call $\underline{u}(x, t)$ is the lower solution of problem (1.1)-(1.3), in $Q_{T}$.

Similarly, a nonnegative function $\bar{u}(x, t) \in C^{2,1}\left(Q_{T}\right) \cap C\left(\bar{Q}_{T}\right)$ is an upper solution if it satisfies (2.1)-(2.3) in the reverse order. We say $u(x, t)$ is a solution of problem (1.1)-(1.3) in $Q_{T}$ if it is both an upper solution and a lower solution of problem (1.1)-(1.3) in $Q_{T}$ which is called classical solution.

The following comparison principle plays a crucial role in our proofs which can be obtained by establishing suitable test function and Gronwall's inequality.

Proposition 2.2 (comparison principle). Suppose that $\underline{u}(x, t)$ and $\bar{u}(x, t)$ are the nonnegative subsolution and supersolution of problem (1.1)-(1.3), respectively. Moreover $\underline{u}(x, 0) \leq \bar{u}(x, 0), \underline{u}(x, 0) \geq$ 0 , and $\bar{u}(x, 0) \geq \delta>0$ in $\bar{\Omega}$, then $\underline{u}(x, t) \leq \bar{u}(x, t)$ in $\bar{Q}_{T}$. 
Proof. Let $\psi(x, t) \in C^{2,1}\left(Q_{T}\right)$ be a nonnegative function with $\psi=0$ on $S_{T}$. Multiplying the inequality in (2.1) by $\psi(x, t)$ and integrating it on $Q_{T}$, we get

$$
\begin{aligned}
\iint_{Q_{T}} \underline{u}_{t} \psi d x d t \leq & \iint_{Q_{T}} \underline{u} \psi_{t}+\underline{u}^{m} \Delta \psi+\psi\left(\underline{u}^{p} \int_{\Omega} \underline{u}^{q} d y-k \underline{u}^{r}\right) d x d t \\
& -\int_{0}^{t} \int_{\partial \Omega} \frac{\partial \psi}{\partial n}\left(\int_{\Omega} f(x, y) \underline{u} d y\right)^{m} d S d t
\end{aligned}
$$

and similarly, the upper solution satisfies the reversed inequality,

$$
\begin{aligned}
\iint_{Q_{T}} \bar{u}_{t} \psi d x d t \leq & \iint_{Q_{T}} \bar{u} \psi_{t}+\bar{u}^{m} \Delta \psi+\psi\left(\bar{u}^{p} \int_{\Omega} \bar{u}^{q} d y-k \bar{u}^{r}\right) d x d t \\
& -\int_{0}^{t} \int_{\partial \Omega} \frac{\partial \psi}{\partial n}\left(\int_{\Omega} f(x, y) \bar{u} d y\right)^{m} d S d t .
\end{aligned}
$$

Let $\omega(x, t)=\underline{u}(x, t)-\bar{u}(x, t)$, we have

$$
\begin{aligned}
\iint_{Q_{T}} \omega_{t} \psi d x d t \leq & \iint_{Q_{T}}\left[\psi_{t}+\Phi_{1}(x, t) \Delta \psi+\left(\Phi_{2}(x, t) \int_{\Omega} \underline{u}^{q} d y-k \Phi_{3}(x, t)\right) \psi\right] \omega d x d t \\
& +\iint_{Q_{T}} \bar{u}^{p} \psi\left(\int_{\Omega} \Phi_{4} \omega d y\right) d x d t-\int_{0}^{t} \int_{\partial \Omega} \frac{\partial \psi}{\partial n} m \xi^{m-1}\left(\int_{\Omega} f(x, y) \omega d y\right) d S d t
\end{aligned}
$$

where

$$
\begin{gathered}
\Phi_{1}(x, t)=\int_{0}^{1} m(\theta \underline{u}(x, t)+(1-\theta) \bar{u}(x, t))^{m-1} d \theta, \\
\Phi_{2}(x, t)=\int_{0}^{1} p(\theta \underline{u}(x, t)+(1-\theta) \bar{u}(x, t))^{p-1} d \theta, \\
\Phi_{3}(x, t)=\int_{0}^{1} r(\theta \underline{u}(x, t)+(1-\theta) \bar{u}(x, t))^{r-1} d \theta, \\
\Phi_{4}(x, t)=\int_{0}^{1} q(\theta \underline{u}(x, t)+(1-\theta) \bar{u}(x, t))^{q-1} d \theta,
\end{gathered}
$$

and $\xi$ is a function between $\int_{\Omega} f(x, y) \underline{u} d y$ and $\int_{\Omega} f(x, y) \bar{u} d y$. Noticing that $\underline{u}(x, t)$ and $\bar{u}(x, t)$ are bounded functions, it follows from $m>1, p \geq 1, q \geq 1$, and $r \geq 1$ that $\Phi_{i}(i=1,2,3,4)$ are bounded nonnegative functions. If $0 \leq p<1$ or $0<q<1$, we have $\Phi_{2} \leq \delta^{p-1}$ and $\Phi_{4} \leq \delta^{q-1}$ by the condition that $\underline{u}(x, 0) \geq 0$ or $\bar{u}(x, 0) \geq \delta>0$. Thus, we may choose an appropriate $\psi(x, t)$ as in [17, p. 118-123] to obtain

$$
\int_{\Omega} \omega_{+} d x \leq C_{1} \int_{\Omega} \omega(x, 0)_{+} d x+C_{2} \iint_{Q_{T}} \omega(x, t) d x d t
$$


where $\omega_{+}=\max \{\omega, 0\}$ and $C_{1}, C_{2}>0$. It follows from $\underline{u}(x, 0) \leq \bar{u}(x, 0)$ that

$$
\int_{\Omega} \omega_{+} d x \leq C_{2} \iint_{Q_{T}} \omega(x, t) d x d t
$$

By Gronwall's inequality, we know that $(\underline{u}-\bar{u})_{+} \leq 0$, that is, $\underline{u}(x, t) \leq \bar{u}(x, t)$ in $Q_{T}$.

For $x \in \partial \Omega, y \in \Omega, t>0$,

$$
\underline{u}-\bar{u} \leq \int_{\Omega} f(x, y)(\underline{u}-\bar{u}) d y \leq 0 .
$$

This completes the proof.

Next, we state the local existence and uniqueness theorem without proof.

Theorem 2.3 (local existence and uniqueness). Suppose that the nonnegative initial data $u_{0}(x) \in$ $C^{2+\alpha}(\Omega) \cap C(\bar{\Omega})(0<\alpha<1)$ satisfies the compatibility condition. Then, there exists a constant $T^{*}>0$ such that the problem (1.1)-(1.3) admits nonnegative solution $u(x, t) \in C^{2,1}\left(Q_{T}\right) \cap C\left(\bar{\Omega}_{T}\right)$ for each $T<T^{*}$. Furthermore, either $T^{*}=\infty$ or

$$
\lim _{t \rightarrow T^{*}} \sup \|u(x, t)\|_{\infty}=\infty
$$

Remark 2.4. The existence of local nonnegative solutions in time to problem (1.1)-(1.3) can be obtained by using the fixed point theorem (see [18]) or the regular theory to get the suitable estimate in a standard limiting process (see $[19,20]$ ). By the previous comparison principle, we can get the uniqueness of solution to the problem (1.1)-(1.3) in the case of $p+q \geq 1, r \geq 1$.

\section{Global Existence and Blowup of Solutions}

Comparing problems with the general homogeneous Dirichlet boundary condition, the existence of weight function on the boundary has a great influence on the global and nonglobal existence of solutions.

Proof of Theorem 1.1. Consider the following problem:

$$
v^{\prime}(t)=|\Omega| v^{p+q}-k v^{r}, \quad v(0)=v_{0}
$$

As $p+q>r$, we know that $v^{p+q}+1>v^{r}$, and $|\Omega| v^{p+q}-k v^{r} \geq(|\Omega|-k) v^{p+q}-k$. Therefore, the solution of (3.1) is an upper solution of the following problem:

$$
v^{\prime}(t)=(|\Omega|-k) v^{p+q}-k, \quad v(0)=v_{0}
$$

When $|\Omega|>k$ and $p+q>1$, it is known that the solution to the problem (3.2) blows up in finite time if $v_{0}>(k /(|\Omega|-k))^{1 /(p+q)}$. 
It is obvious that the solution of problem (3.1) is a lower solution of problem (1.1)(1.3) when $\int_{\Omega} f(x, y) d y \geq 1$ and $u_{0}(x)>v_{0}$. By Proposition 2.2, $u(x, t)$ is a blow-up solution of problem (1.1)-(1.3).

Proof of Theorem 1.3. (1) The case of $p+q>r$. Let $\bar{u}=(k /|\Omega|)^{1 /(p+q-r)}$. It is easy to show that if $\int_{\Omega} f(x, y) d y<1$ and $u_{0}(x)<(k /|\Omega|)^{1 /(p+q-r)}, \bar{u}(x, t)$ is the upper solution of problem (1.1)-(1.3), then we can draw the conclusion.

(2) The case of $p+q \geq \max \{m, r\}$. We need to establish a self-similar blow-up solution in order to prove the blow-up result. We first suppose that $\omega \in C^{1}(\bar{\Omega}), \omega(x) \geq 0$, and $\omega(x)$ is not identically zero, and $\left.\omega(x)\right|_{\partial \Omega}=0$. Without loss of generality, we assume that $0 \in \Omega$ and $\omega(0)>0$.

Let $\underline{u}(x, t)=(T-t)^{-\gamma} V^{1 / m}(\xi), V(\xi)=\left(1-\xi^{2} / 2 A\right)_{+}$, and $\xi=|x|(T-t)^{-\mu}$, where $A>$ $1,0<T<\overline{1}, \gamma$ and $\mu>0$. We know that

$$
\operatorname{supp} \underline{u}_{+}(\cdot, t)=\overline{B\left(0, R(T-t)^{\mu}\right)} \subset \overline{B\left(0, R T^{\mu}\right)} \subset \Omega,
$$

for sufficiently small $T>0$ and $R=\sqrt{A(A+2)}$. Calculating the derivative of $\underline{u}$, we obtain

$$
\begin{aligned}
\underline{u}_{t}= & \frac{m \gamma V^{1 / m}(\xi)+\mu \xi V^{\prime}(\xi) V^{(1-m) / m}}{m(T-t)^{\gamma+1}}, \\
\underline{u}^{p} \int_{\Omega} \underline{u}^{q}(y, t) d y & =\frac{\Delta \underline{u}^{m}=-\frac{N}{A(T-t)^{m \gamma+2 \mu}}}{(T-t)^{(p+q) \gamma}} \int_{B\left(0, R(T-t)^{\mu}\right)} V^{q / m}\left(\frac{|x|}{(T-t)^{\mu}}\right) d x \\
& \geq \frac{M}{(T-t)^{(p+q) \gamma-N \mu}},
\end{aligned}
$$

where $M=\int_{B(0, R)} V^{q / m}(|\xi|) d \xi>0$.

It is easy to see that $V^{\prime}(\xi) \leq 0$ and $V<1$, and

$$
\begin{aligned}
\underline{u}_{t} & -\Delta \underline{u}^{m}-\underline{u}^{p} \int_{\Omega} \underline{u}^{q}(y, t) d y+k \underline{u}^{r} \\
\leq & \frac{m \gamma V^{1 / m}(\xi)+\mu \xi V^{\prime}(\xi) V^{(1-m) / m}}{m(T-t)^{\gamma+1}}+\frac{N}{A(T-t)^{m \gamma+2 \mu}} \\
& -\frac{M}{(T-t)^{(p+q) \gamma-N \mu}}+k(T-t)^{-r \gamma} V^{r / m}(\xi) \\
\leq & \frac{\gamma}{(T-t)^{\gamma+1}}+\frac{N}{A(T-t)^{m \gamma+2 \mu}}-\frac{M}{(T-t)^{(p+q) \gamma-N \mu}}+\frac{k}{(T-t)^{r \gamma}} .
\end{aligned}
$$


Since $p+q \geq m>1$, choosing $\gamma>0$ such that $m<(1+\gamma) / \gamma<p+q$, and $\mu$ is sufficiently small such that

$$
\mu<\min \left\{\frac{(p+q-1) \gamma-1}{N}, \frac{(p+q-r) \gamma}{N}, \frac{p+q-m}{2+N}\right\}
$$

Then, for small enough $T>0$, we have

$$
\underline{u}_{t}-\Delta \underline{u}^{m}-\underline{u}^{p} \int_{\Omega} \underline{u}^{q}(y, t) d y+k \underline{u}^{r} \leq 0
$$

If $x \in \partial \Omega, \omega(0)>0$, and $\omega$ is continuous, it is known that there exist positive $\varepsilon$ and $\rho$ such that $\omega \geq \varepsilon$ for $x \in B(0, \rho)$. We can get $B\left(0, R T^{\sigma}\right) \subset B(0, \rho) \subset \Omega$ if $T$ is small enough. Then, $\underline{u} \leq \int_{\Omega} f(x, y) \underline{u} d y$ on $\partial \Omega \times(0, T)$. It follows from (3.3) that $\underline{u}(x, 0) \leq K_{0} \omega(x)$ for sufficiently large $K_{0}$. Therefore, one can observe that the solution to (1.1)-(1.3) exists no later than $t=T$ provided that $u_{0} \geq K_{0} \omega(x)$. This implies that the solution blows up in finite time for large enough initial data.

Proof of Theorem 1.4. Suppose that $\lambda_{1}>0$ is the first eigenvalue of $-\Delta$ with homogeneous Dirichlet boundary condition, and $\phi(x)$ is the corresponding eigenfunction. Let $M \int_{\Omega}(1 /(\phi(y)+\varepsilon)) d y \leq 1$ for some $0<\varepsilon<1$, where $M=\max _{x \in \partial \Omega, y \in \bar{\Omega}} f(x, y)$.

Now, we assume that $u^{m}=v$, then (1.1)-(1.3) becomes that

$$
\begin{gathered}
\left(v^{n}\right)_{t}-\Delta v=v^{n p} \int_{\Omega} v^{q n} d y-k v^{n r}, \quad x \in \Omega, t>0, \\
v(x, t)=\left(\int_{\Omega} f(x, y) v^{n}(y, t) d y\right)^{m}, \quad x \in \partial \Omega, t>0, \\
v(x, 0)=v_{0}(x)=u_{0}^{m}(x), \quad x \in \bar{\Omega},
\end{gathered}
$$

where $n=1 / m$.

Set $\bar{v}(x, t)=C e^{\gamma t / n} /(\phi(x)+\varepsilon)$, where $C$ is determined later, then it follows that

$$
\begin{gathered}
\left(\bar{v}^{n}\right)_{t}=\frac{C^{n} \gamma e^{\gamma t}}{(\phi(x)+\varepsilon)^{n}} \\
\nabla \bar{v}=\frac{-C e^{\gamma t / n} \nabla \phi(x)}{(\phi(x)+\varepsilon)^{2}}=-\frac{\nabla \phi}{\phi(x)+\varepsilon} \bar{v} \\
\Delta \bar{v}=\frac{(\phi+\varepsilon)(-\bar{v} \Delta \phi-\nabla \phi \cdot \nabla \bar{v})+\bar{v} \nabla \phi \cdot \nabla \phi}{(\phi+\varepsilon)^{2}} \\
=\frac{\lambda_{1} \phi \bar{v}-\nabla \phi \cdot \nabla \bar{v}}{\phi+\varepsilon}+\frac{|\nabla \phi|^{2}}{(\phi+\varepsilon)^{2}} \bar{v}=\left(\frac{\lambda_{1} \phi}{\phi+\varepsilon}+\frac{2|\nabla \phi|^{2}}{(\phi+\varepsilon)^{2}}\right) \bar{v} .
\end{gathered}
$$


And, we can get

$$
\begin{aligned}
\left(\bar{v}^{n}\right)_{t}-\Delta \bar{v}-v^{p n} \int_{\Omega} v^{q n} d y-k v^{n r} \\
=\frac{C^{n} \gamma e^{\gamma t}}{(\phi(x)+\varepsilon)^{n}}-\left(\frac{\lambda_{1} \phi}{\phi+\varepsilon}+\frac{2|\nabla \phi|^{2}}{(\phi+\varepsilon)^{2}}\right) \bar{v} \\
\quad-\frac{\left(C e^{\gamma t / n}\right)^{n(p+q)}}{(\phi+\varepsilon)^{n p}} \int_{\Omega} \frac{1}{(\phi+\varepsilon)^{n q} d y}+k\left(C e^{\gamma t / n}\right)^{n r}(\phi+\varepsilon)^{n r} \\
=\left[\left(\frac{C}{\phi+\varepsilon}\right)^{n-1} \gamma e^{(\gamma-\gamma / n) t}-\left(\frac{\lambda_{1} \phi}{\phi+\varepsilon}+\frac{2|\nabla \phi|^{2}}{(\phi+\varepsilon)^{2}}\right)\right] \bar{v} \\
\quad-\frac{\left(C e^{\gamma t / n}\right)^{n(p+q)}}{(\phi+\varepsilon)^{n p}} \int_{\Omega} \frac{1}{(\phi+\varepsilon)^{n q} d y}+k C^{n r} e^{\gamma r t}(\phi+\varepsilon)^{n r} .
\end{aligned}
$$

Choosing $C>\max \left\{\sup _{\bar{\Omega}}\left[\left((\phi+\varepsilon)^{n(r-p)} / k\right) \int_{\Omega}\left(1 /(\phi+\varepsilon)^{n q} d y\right)\right]^{1 / n(r-p-q)}, \sup (\phi+\varepsilon) u_{0}(x)\right\}$ and the proper $\lambda_{1}$ such that

$$
r\left(\frac{C}{\phi+\varepsilon}\right)^{n-1}>\frac{\lambda_{1} \phi}{\phi+\varepsilon}+\frac{2|\nabla \phi|^{2}}{(\phi+\varepsilon)^{2}}
$$

then

$$
\left(\bar{v}^{n}\right)_{t}-\Delta \bar{v}-v^{p n} \int_{\Omega} v^{q n} d y-k v^{n r} \geq 0
$$

For $x \in \partial \Omega, t>0$,

$$
\bar{v}(x, t)=\frac{C e^{\gamma t / n}}{\varepsilon} \geq \int_{\Omega} \frac{C e^{\gamma t / n} M}{\phi(y)+\varepsilon} d y \geq \int_{\Omega} f(x, y) \bar{v} d y,
$$

so we can obtain that $\bar{v}(x, t)$ is the upper solution of problem $(*)-(* * *)$. From Proposition 2.2, we know that there exists global solution of problem $(*)-(* * *)$. Since the problem (1.1)-(1.3) has the same solution with problem $(*)-(* * *)$. We know that there exists a global solution of problem (1.1)-(1.3). This completed the proof.

Proof of Theorem 1.5. Suppose that $\Phi(x)$ solves the following problem:

$$
\begin{gathered}
-\Delta \Phi=\delta, \quad x \in \Omega, \\
\Phi(x)=0, \quad x \in \partial \Omega
\end{gathered}
$$

where $\delta$ is a positive constant such that $0 \leq \Phi(x) \leq 1$. Then, let $M_{1}=\max _{x \in \bar{\Omega}} \Phi(x) \leq 1$. 
Set $\omega(x, t)=A\left(\rho^{m} /\left(1-\rho^{m}\right)+\Phi(x) / M_{1}\right)^{1 / m}$, here $A>0$ will be determined later and $0<\rho<1$,

$$
\begin{aligned}
\omega_{t}- & \Delta \omega^{m}-\omega^{p} \int_{\Omega} \omega^{q}(y, t) d y+k \omega^{r} \\
= & \frac{A^{m} \delta}{M_{1}}-A^{p+q}\left(\frac{\rho^{m}}{1-\rho^{m}}+\frac{\Phi(x)}{M_{1}}\right)^{p / m} \int_{\Omega}\left(\frac{\rho^{m}}{1-\rho^{m}}+\frac{\Phi(x)}{M_{1}}\right)^{q / m} d x \\
& +k A^{p+q}\left(\frac{\rho^{m}}{1-\rho^{m}}+\frac{\Phi(x)}{M_{1}}\right)^{(p+q) / m} \\
\geq & \frac{A^{m} \delta}{M_{1}}-A^{p+q}|\Omega|\left(\frac{\rho^{m}}{1-\rho^{m}}+\frac{\Phi(x)}{M_{1}}\right)^{(p+q) / m}+k A^{p+q}\left(\frac{\rho^{m}}{1-\rho^{m}}+\frac{\Phi(x)}{M_{1}}\right)^{(p+q) / m} \\
= & \frac{A^{m} \delta}{M_{1}}+A^{p+q}(k-|\Omega|)\left(\frac{\rho^{m}}{1-\rho^{m}}+\frac{\Phi(x)}{M_{1}}\right)^{(p+q) / m} .
\end{aligned}
$$

It is obvious that the global existence result holds for $k \geq|\Omega|$. For $k<|\Omega|$, since $\Phi(x) / M_{1} \leq 1$, we know that $\rho^{m} /\left(1-\rho^{m}\right)+\Phi(x) / M_{1} \leq \rho^{m} /\left(1-\rho^{m}\right)+1=1 /\left(1-\rho^{m}\right)$, then

$\omega_{t}-\Delta \omega^{m}-\omega^{p} \int_{\Omega} \omega^{q}(y, t) d y+k \omega^{r} \geq \frac{A^{m} \delta}{M_{1}}-A^{p+q}(|\Omega|-k)\left(\frac{1}{1-\rho^{m}}\right)^{(p+q) / m}$.

(1) If $m>p+q$, choosing $A=\max \left\{\max \left|u_{0}(x)\right|,(|\Omega|-k)(M / \delta)\left(1 /\left(1-\rho^{m}\right)\right)^{(p+q) / m}\right\}$, we have

$$
\omega_{t}-\Delta \omega^{m}-\omega^{p} \int_{\Omega} \omega^{q}(y, t) d y+k \omega^{r} \geq 0
$$

(2) If $m=p+q$, selecting $|\Omega|<k+\left(\delta / M_{1}\right)\left(1-\rho^{m}\right)$, and $A=\max u_{0}(x)$, we get

$$
\omega_{t}-\Delta \omega^{m}-\omega^{p} \int_{\Omega} \omega^{q}(y, t) d y+k \omega^{r} \geq 0
$$

(3) If $m<p+q$, we choose $\max u_{0}(x) \leq A \leq(|\Omega|-k)\left(M_{1} / \delta\right)\left(1 /\left(1-\rho^{m}\right)\right)^{-(p+q) / m}$ such that

$$
\omega_{t}-\Delta \omega^{m}-\omega^{p} \int_{\Omega} \omega^{q}(y, t) d y+k \omega^{r} \geq 0
$$

For $x \in \partial \Omega$,

$$
\begin{aligned}
\omega(x, t) & =A\left(\frac{\rho^{m}}{1-\rho^{m}}\right)^{1 / m}=A\left(\frac{\rho^{m}}{1-\rho^{m}}+1\right)^{1 / m} \rho \\
& \geq A\left(\frac{\rho^{m}}{1-\rho^{m}}+\frac{\Phi(x)}{M_{1}}\right)^{1 / m} \int_{\Omega} f(x, y) d y \\
& =\int_{\Omega} \omega(y, t) f(x, y) d y .
\end{aligned}
$$

Through the previous discussion, we know that the global existence results hold. 
For the blow-up case of $m<p+q$, it holds clearly from the second part of the proof of Theorem 1.3.

\section{Blow-Up Rate Estimates}

Next, we will get the following precise blow-up rate estimates for slow diffusion case under some suitable conditions.

Let $v=u^{m}$, we just need to consider the problem $(*)-(* * *)$, and let $p n=p_{1}, q n=q_{1}$, and $r n=r_{1}$, then $(*)$ becomes

$$
\left(v^{n}\right)_{t}=\Delta v+v^{p_{1}} \int_{\Omega} v^{q_{1}}(y, t) d y-k v^{r_{1}}, \quad x \in \Omega, t>0
$$

Suppose that $v(x, t)$ is the blow-up solution of problem $(*)-(* * *)$ in finite time $T$, and set $V(t)=\max _{x \in \bar{\Omega}} v(x, t)$.

Proof of Theorem 1.6. (1) We can easily know that $V(t)$ is Lip continuous and differential almost everywhere,

$$
\left(V^{n}\right)_{t} \leq V^{p_{1}} \int_{\Omega} V^{q_{1}} d y-k V^{r_{1}} \leq V^{p_{1}+q_{1}}|\Omega|-k V^{r_{1}} \leq V^{p_{1}+q_{1}}|\Omega|
$$

Then, it follows that

$$
V_{t} \leq \frac{1}{n}|\Omega| V^{p_{1}+q_{1}+1-n}
$$

Integrating it over $(t, T)$, we get

$$
V(t) \geq\left[\frac{1}{n}|\Omega|\left(p_{1}+q_{1}-n\right)\right]^{-1 /\left(p_{1}+q_{1}-n\right)}(T-t)^{-1 /\left(p_{1}+q_{1}-n\right)},
$$

then

$$
u(x, t) \geq c(T-t)^{-1 /(p+q-1)},
$$

where $c=[|\Omega|(p+q-1)]^{-1 /(p+q-1)}$.

(2) Next, we set $J=v_{t}-\delta v^{p_{1}+q_{1}+1-n}$, where $\delta>0$, then

$$
\begin{aligned}
J_{t}= & v_{t t}-\delta\left(p_{1}+q_{1}+1-n\right) v^{p_{1}+q_{1}-n} v_{t} \\
= & \frac{1-n}{n} v^{-n}\left(v^{n}\right)_{t} v_{t}+\frac{1}{n} v^{1-n}\left(\Delta v+v^{p_{1}} \int_{\Omega} v^{q_{1}} d y-k v^{r_{1}}\right)_{t}-\delta\left(p_{1}+q_{1}+1-n\right) v^{p_{1}+q_{1}-n} v_{t} \\
= & (1-n) v^{-1}\left(v_{t}\right)^{2}+\frac{1}{n} v^{1-n} \Delta v_{t}+\frac{p_{1}}{n} v^{p_{1}-n} v_{t} \int_{\Omega} v^{q_{1}} d y+\frac{q_{1}}{n} v^{p_{1}+1-n} \int_{\Omega} v^{q_{1}-1} v_{t} d y \\
& -\frac{k r_{1}}{n} v^{r_{1}-n} v_{t}-\delta\left(p_{1}+q_{1}+1-n\right) v^{p_{1}+q_{1}-n} v_{t}
\end{aligned}
$$


Abstract and Applied Analysis

$$
\begin{aligned}
J_{t}- & \frac{v^{1-n}}{n} \Delta J-\left[2 \delta(1-n) v^{p_{1}+q_{1}-n}+\frac{p_{1} v^{p_{1}-n}}{n} \int_{\Omega} v^{q_{1}} d y-\frac{k r_{1}}{n} v^{r_{1}-n}\right] J-\frac{q_{1} v^{p_{1}+1-n}}{n} \int_{\Omega} v^{q_{1}-1} J d y \\
= & (1-n) v^{-1} J^{2}+\delta^{2}(1-n) v^{2 p_{1}+2 q_{1}-2 n+1}+\left[\frac{p_{1} \delta}{n}-\frac{\delta}{n}\left(p_{1}+q_{1}+1-n\right)\right] v^{2 p_{1}+q_{1}-2 n+1} \int_{\Omega} v^{q_{1}} d y \\
& +\left[\frac{k \delta}{n}\left(p_{1}+q_{1}+1-n\right)-\frac{k r_{1} \delta}{n}\right] v^{p_{1}+q_{1}+r_{1}-2 n+1}+\frac{\delta}{n}\left(p_{1}+q_{1}-n+1\right)\left(p_{1}+q_{1}-n\right)|\nabla v|^{2} \\
& +\frac{q_{1} \delta}{n} v^{p_{1}+1-n} \int_{\Omega} v^{p_{1}+2 q_{1}-n} d y \\
\geq & \delta^{2}(1-n) v^{2 p_{1}+2 q_{1}-2 n+1} \\
& +\frac{\delta}{n}\left(q_{1}+1-n\right) v^{2 p_{1}+q_{1}-2 n+1} \int_{\Omega} v^{q_{1}} d y+\frac{k \delta}{n}\left(p_{1}+q_{1}+1-n-r_{1}\right) v^{p_{1}+q_{1}+r_{1}-2 n+1} \\
& +\frac{q_{1} \delta}{n} v^{p_{1}+1-n} \int_{\Omega} v^{p_{1}+2 q_{1}-n} d y \\
= & \delta^{2}(1-n) v^{2 p_{1}+2 q_{1}-2 n+1}+\frac{\delta}{n} v^{p_{1}-n+1}\left[q_{1} \int_{\Omega} v^{p_{1}+2 q_{1}-n} d y-\left(q_{1}+1-n\right) v^{p_{1}+q_{1}-n} \int_{\Omega} v^{q_{1}} d y\right] \\
& +\frac{k \delta}{n}\left(p_{1}+q_{1}+1-n-r_{1}\right) v^{p_{1}+q_{1}+r_{1}-2 n+1} .
\end{aligned}
$$

Since $q_{1} /\left(p_{1}+2 q_{1}-n\right)+\left(p_{1}+q_{1}-n\right) /\left(p_{1}+2 q_{1}-n\right)=1$, by Hölder's inequality, we know that

$$
\int_{\Omega} v^{q_{1}} d y \leq\left(\int_{\Omega} v^{p_{1}+2 q_{1}-n}\right)^{q_{1} /\left(p_{1}+2 q_{1}-n\right)}
$$

and by Young inequality, we have

$$
\begin{aligned}
& v^{p_{1}+q_{1}-n}\left(\int_{\Omega} v^{p_{1}+2 q_{1}-n}\right)^{q_{1} /\left(p_{1}+2 q_{1}-n\right)} \\
& \quad \leq \frac{p_{1}+q_{1}-n}{p_{1}+2 q_{1}-n}\left(v^{p_{1}+q_{1}-n}\right)^{\left(p_{1}+2 q_{1}-n\right) /\left(p_{1}+q_{1}-n\right)}+\frac{q_{1}}{p_{1}+2 q_{1}-n} \int_{\Omega} v^{p_{1}+2 q_{1}-n} \\
& \quad=\frac{p_{1}+q_{1}-n}{p_{1}+2 q_{1}-n} v^{p_{1}+2 q_{1}-n}+\frac{q_{1}}{p_{1}+2 q_{1}-n} \int_{\Omega} v^{p_{1}+2 q_{1}-n} .
\end{aligned}
$$

Since $p_{1}+q_{1}>r_{1}$, we get $p_{1}+2 q_{1}-n>q_{1}+1-n$ and $p_{1}+q_{1}-r_{1}>0$, then by (4.7)-(4.8),

$$
\begin{aligned}
J_{t} & -\frac{v^{1-n}}{n} \Delta J-\left[2 \delta(1-n) v^{p_{1}+q_{1}-n}+\frac{p_{1} v^{p_{1}-n}}{n} \int_{\Omega} v^{q_{1}} d y-\frac{k r_{1}}{n} v^{r_{1}-n}\right] J-\frac{q_{1} v^{p_{1}+1-n}}{n} \int_{\Omega} v^{q_{1}-1} J d y \\
& \geq \delta^{2}(1-n) v^{2 p_{1}+2 q_{1}-2 n+1}+\frac{k \delta}{n}\left(p_{1}+q_{1}+1-n-r_{1}\right) v^{p_{1}+q_{1}+r_{1}-2 n+1}-\frac{\delta p_{1}+q_{1}-n}{n} v^{2 p_{1}+2 q_{1}-2 n+1} \\
& \geq\left[\delta(1-n)-\frac{p_{1}+q_{1}-n}{n}\right] \delta v^{2 p_{1}+2 q_{1}-2 n+1} .
\end{aligned}
$$


Choosing $\delta \geq\left(p_{1}+q_{1}-n\right) / n(1-n)$, such that

$$
\begin{aligned}
& J_{t}-\frac{v^{1-n}}{n} \Delta J-\left[2 \delta(1-n) v^{p_{1}+q_{1}-n}+\frac{p_{1} v^{p_{1}-n}}{n} \int_{\Omega} v^{q_{1}} d y-\frac{k r_{1}}{n} v^{r_{1}-n}\right] J-\frac{q_{1} v^{p_{1}+1-n}}{n} \int_{\Omega} v^{q_{1}-1} J d y \\
& \quad \geq 0 .
\end{aligned}
$$

For $(x, t) \in S_{T}$, because of $v_{t}=J+\delta v^{p_{1}+q_{1}+1-n}$, we then have

$$
\begin{aligned}
J & =v_{t}-\delta v^{p_{1}+q_{1}+1-n} \\
& =m\left(\int_{\Omega} f(x, y) v^{1 / m} d y\right)^{m-1} \int_{\Omega} f(x, y)\left(v^{1 / m}\right)_{t} d y-\delta\left(\int_{\Omega} f(x, y) v^{1 / m} d y\right)^{m\left(p_{1}+q_{1}+1-n\right)} \\
& =\left(\int_{\Omega} f(x, y) v^{1 / m} d y\right)^{m-1}\left[m \int_{\Omega} f(x, y)\left(v^{1 / m}\right)_{t} d y-\delta\left(\int_{\Omega} f(x, y) v^{1 / m} d y\right)^{p+q}\right] \\
& =\left(\int_{\Omega} f(x, y) v^{1 / m} d y\right)^{m-1}\left[\int_{\Omega} f(x, y) v^{(1-m) / m} J d y\right. \\
& \left.+\delta\left(\int_{\Omega} f(x, y) v^{(p+q) / m} d y-\left(\int_{\Omega} f(x, y) v^{1 / m} d y\right)^{p+q}\right)\right]
\end{aligned}
$$

Noticing that $0<F(x)=\int_{\Omega} f(x, y) d y \leq 1$ for $x \in \partial \Omega, p+q>\max \{m, r\}$, and applying Jensen's inequality to the last part in the previous inequality, we can get

$$
\begin{aligned}
& \int_{\Omega} f(x, y) v^{(p+q) / m} d y-\left(\int_{\Omega} f(x, y) v^{1 / m} d y\right)^{p+q} \\
& \quad \geq F(x)\left(\int_{\Omega} f(x, y) v^{1 / m} \frac{d y}{F(x)}\right)^{p+q}-\left(\int_{\Omega} f(x, y) v^{1 / m} d y\right)^{p+q} .
\end{aligned}
$$

Hence, we can get

$$
J \geq\left(\int_{\Omega} f(x, y) v^{1 / m} d y\right)^{m-1} \int_{\Omega} f(x, y) v^{(1-m) / m} J d y \geq 0
$$

Since $u_{0}(x)$ satisfies the conditions $\left(\mathrm{C}_{1}\right)-\left(\mathrm{C}_{2}\right)$ and $v=u^{m}$,

$$
\begin{aligned}
J(x, t) & =m u^{m-1} u_{t}-\delta u^{m\left(p_{1}+q_{1}+1-n\right)} \\
& =m u^{m-1}\left(\Delta u^{m}+u^{p} \int_{\Omega} u^{q}(y, t) d y-k u^{r}-\delta^{\prime} u^{p+q}\right),
\end{aligned}
$$

where $\delta^{\prime}=\delta / m$, then $J(x, 0) \geq 0$. Combining (4.10)-(4.13), we can know that $J(x, t) \geq 0$ for $(x, t) \in \bar{Q}_{T}$, that is, $v_{t} \geq \delta v^{p_{1}+q_{1}+1-n}$. 
Integrating it over $(t, T)$, we have

$$
v \leq\left[\delta\left(p_{1}+q_{1}-n\right)\right]^{-1 /\left(p_{1}+q_{1}-n\right)}(T-t)^{-1 /\left(p_{1}+q_{1}-n\right)},
$$

then it follows that

$$
u(x, t) \leq C(T-t)^{-1 /(p+q-1)},
$$

where $C=[\delta(p+q-1) / m]^{-1 /(p+q-1)}$. This completed the proof.

\section{Acknowledgments}

This work is supported by the Natural Science Foundation of Shandong Province of China (ZR2012AM018). The authors would like to deeply thank all the reviewers for their insightful and constructive comments.

\section{References}

[1] J. Bebernes and D. Eberly, Mathematical Problems from Combustion Theory, vol. 83 of Applied Mathematical Sciences, Springer, New York, NY, USA, 1989.

[2] C. V. Pao, Nonlinear Parabolic and Elliptic Equations, Plenum Press, New York, NY, USA, 1992.

[3] J. Furter and M. Grinfeld, "Local versus nonlocal interactions in population dynamics," Journal of Mathematical Biology, vol. 27, no. 1, pp. 65-80, 1989.

[4] Calsina, C. Perelló, and J. Saldaña, "Non-local reaction-diffusion equations modelling predator-prey coevolution," Publicacions Matemàtiques, vol. 38, no. 2, pp. 315-325, 1994.

[5] W. Allegretto, G. Fragnelli, P. Nistri, and D. Papini, "Coexistence and optimal control problems for a degenerate predator-prey model," Journal of Mathematical Analysis and Applications, vol. 378, no. 2, pp. 528-540, 2011.

[6] W. A. Day, "Extensions of a property of the heat equation to linear thermoelasticity and other theories," Quarterly of Applied Mathematics, vol. 40, no. 3, pp. 319-330, 1982/83.

[7] W. A. Day, "A decreasing property of solutions of parabolic equations with applications to thermoelasticity," Quarterly of Applied Mathematics, vol. 40, no. 4, pp. 468-475, 1982/83.

[8] A. Friedman, "Monotonic decay of solutions of parabolic equations with nonlocal boundary conditions," Quarterly of Applied Mathematics, vol. 44, no. 3, pp. 401-407, 1986.

[9] C. V. Pao, "Asymptotic behavior of solutions of reaction-diffusion equations with nonlocal boundary conditions," Journal of Computational and Applied Mathematics, vol. 88, no. 1, pp. 225-238, 1998.

[10] Y. L. Wang, C. L. Mu, and Z. Y. Xiang, "Blowup of solutions to a porous medium equation with nonlocal boundary condition," Applied Mathematics and Computation, vol. 192, no. 2, pp. 579-585, 2007.

[11] Z. G. Lin and Y. R. Liu, "Uniform blowup profiles for diffusion equations with nonlocal source and nonlocal boundary," Acta Mathematica Scientia, Series B, vol. 24, no. 3, pp. 443-450, 2004.

[12] Z. J. Cui and Z. D. Yang, "Roles of weight functions to a nonlinear porous medium equation with nonlocal source and nonlocal boundary condition," Journal of Mathematical Analysis and Applications, vol. 342, no. 1, pp. 559-570, 2008.

[13] S. N. Zheng and L. H. Kong, "Roles of weight functions in a nonlinear nonlocal parabolic system," Nonlinear Analysis, vol. 68, no. 8, pp. 2406-2416, 2008.

[14] Y. L. Wang, C. L. Mu, and Z. Y. Xiang, "Properties of positive solution for nonlocal reaction diffusion equation with nonlocal boundary," Boundary Value Problems, vol. 2007, Article ID 064579, 12 pages, 2007. 
[15] C. L. Mu, D. M. Liu, and S. M. Zhou, "Properties of positive solutions for a nonlocal reaction-diffusion equation with nonlocal nonlinear boundary condition," Journal of the Korean Mathematical Society, vol. 47, no. 6, pp. 1317-1328, 2010.

[16] M. X. Wang and Y. Wang, "Properties of positive solutions for non-local reaction-diffusion problems," Mathematical Methods in the Applied Sciences, vol. 19, no. 14, pp. 1141-1156, 1996.

[17] J. R. Anderson, "Local existence and uniqueness of solutions of degenerate parabolic equations," Communications in Partial Differential Equations, vol. 16, no. 1, pp. 105-143, 1991.

[18] Y. F. Yin, "On nonlinear parabolic equations with nonlocal boundary condition," Journal of Mathematical Analysis and Applications, vol. 185, no. 1, pp. 161-174, 1994.

[19] L. Damascelli, "Comparison theorems for some quasilinear degenerate elliptic operators and applications to symmetry and monotonicity results," Annales de l'Institut Henri Poincaré, vol. 15, no. 4, pp. 493-516, 1998.

[20] F. C. Li, "Global existence and blow-up of solutions to a nonlocal quasilinear degenerate parabolic system," Nonlinear Analysis, vol. 67, no. 5, pp. 1387-1402, 2007. 


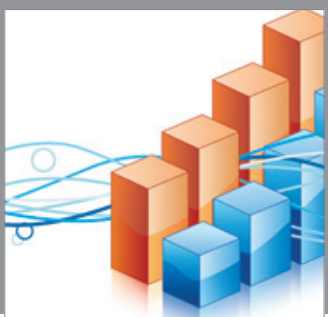

Advances in

Operations Research

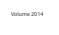

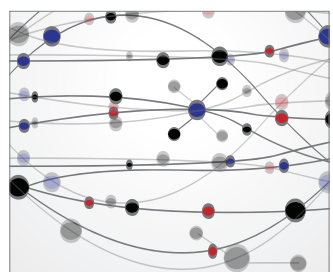

\section{The Scientific} World Journal
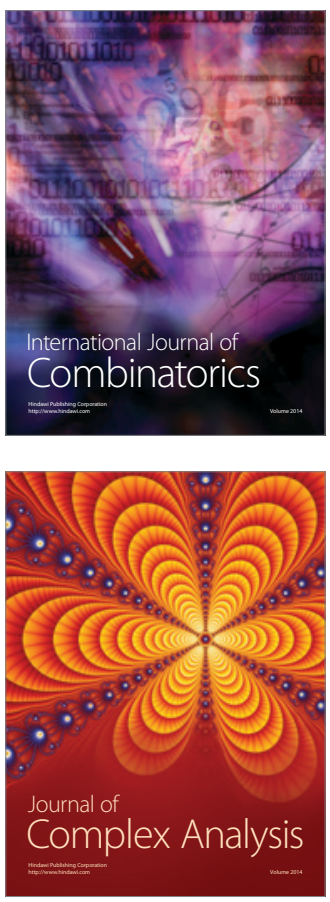

International Journal of

Mathematics and

Mathematical

Sciences
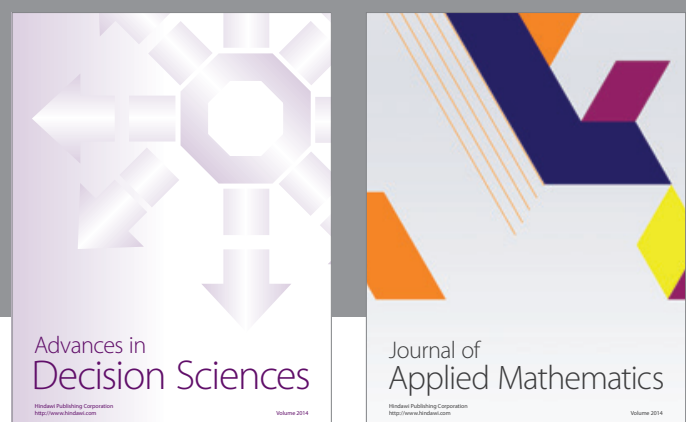

Journal of

Applied Mathematics
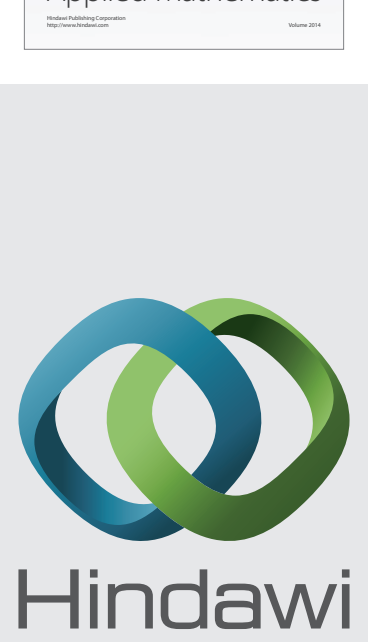

Submit your manuscripts at http://www.hindawi.com
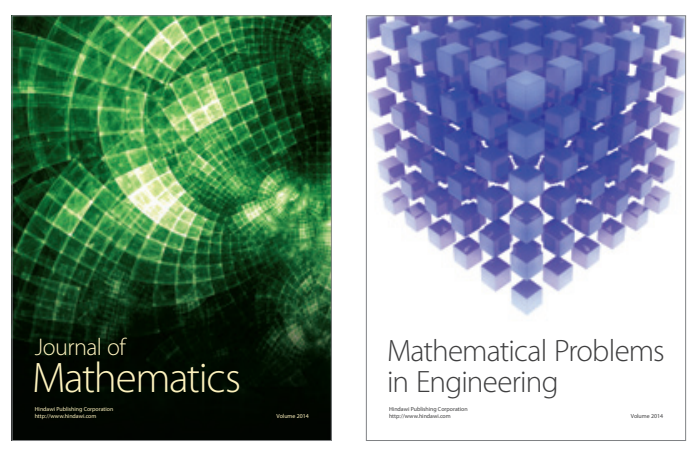

Mathematical Problems in Engineering
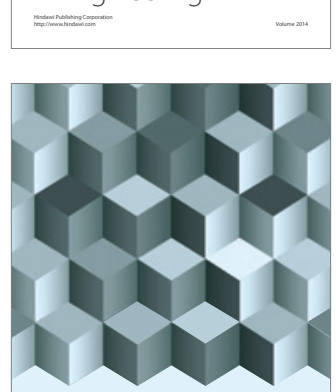

Journal of

Function Spaces
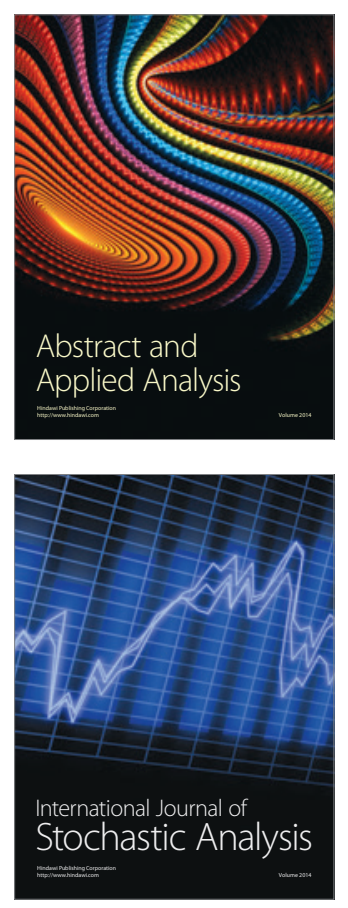

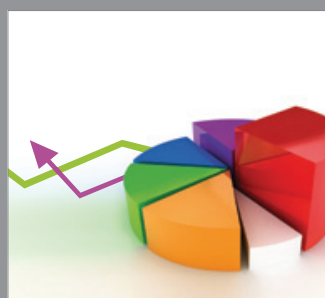

ournal of

Probability and Statistics

Promensencen
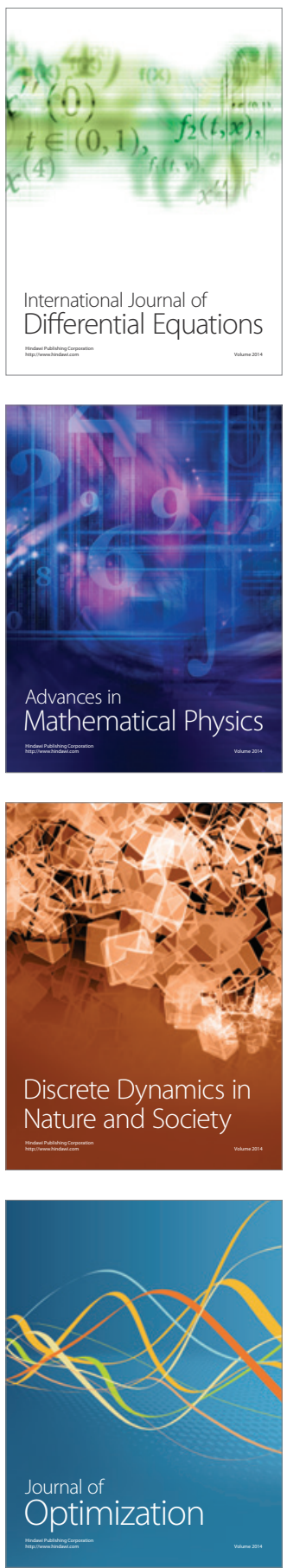